\title{
Study Of The Scattering Of Halo Nuclei Around The Coulomb Barrier
}

L. Acosta ${ }^{\mathrm{a}}$, M. Cubero ${ }^{\mathrm{b}}$, D. Escrig ${ }^{\mathrm{b}}$, J.P. Fernández-García ${ }^{\mathrm{c}}$, J.A. Lay $^{\mathrm{c}}$, A.M. Moro ${ }^{\mathrm{c}}$, M. Rodríguez-Gallardo ${ }^{\mathrm{b}, \mathrm{c}}$, A.M. Sánchez-Benítez ${ }^{\mathrm{a}}$, M. Alcorta ${ }^{\mathrm{b}}$, M.A.G. Álvarez ${ }^{c, d}$, M.V. Andrés ${ }^{c}$, C. Angulo ${ }^{\mathrm{e}}$, M.J.G. Borge ${ }^{\mathrm{b}}$, L. Buchmann ${ }^{\mathrm{f}}$, J. Cabrera ${ }^{\mathrm{e}}$, S. Cherubini ${ }^{\mathrm{g}}$, M.A. Cortés ${ }^{\mathrm{c}}$, P. Demaret ${ }^{\mathrm{e}}$, C.G. Diget ${ }^{\mathrm{h}}$, A. Di Pietro ${ }^{\mathrm{g}}$, J.M. Espino ${ }^{c}$, P. Figuera ${ }^{\mathrm{g}}$, L. M. Fraile ${ }^{\mathrm{i}}$, M. Freer ${ }^{\mathrm{j}}$, B. Fulton ${ }^{\mathrm{h}}$, H.O.U. Fynbo ${ }^{\mathrm{k}}$, D. Galaviz ${ }^{\mathrm{b}, 1}$, J.E. García-Ramos ${ }^{\mathrm{a}}$, M.E. Gómez ${ }^{\mathrm{a}}$, J. Gómez-Camacho ${ }^{\mathrm{c}, \mathrm{d}}$, M. Gulino ${ }^{g}$, O.R. Kakuee ${ }^{\mathrm{m}}$, M. Madurga ${ }^{\mathrm{b}}$, A. Maira-Vidal ${ }^{\mathrm{b}}$, I. Martel ${ }^{\mathrm{a}}$, C. Metelko, A. Musumarra ${ }^{\text {, I. Mukha }}{ }^{c}$, F. Pérez-Bernal ${ }^{\mathrm{a}}$, J. Rahighi ${ }^{\mathrm{m}}$, G.Randisi ${ }^{\mathrm{g}}$, E. Reillo ${ }^{\mathrm{b}}$, J. Rodríguez-Quintero ${ }^{\text {a }}$, K. Rusek ${ }^{\mathrm{n}}$, V. Scuderi ${ }^{\mathrm{g}}$, A. Shotter ${ }^{\mathrm{f}}$, D. Smirnov ${ }^{\mathrm{o}}$,

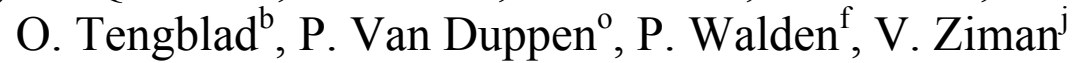

${ }^{a}$ Dpto. de Física Aplicada. Universidad de Huelva E-21071 Huelva, Spain

${ }^{b}$ Instituto de Estructura de la Materia, CSIC, E-28006 Madrid, Spain

${ }^{c}$ Dpto. de Física Atómica, Molecular y Nuclear, Universidad de Sevilla, E-41080 Sevilla, Spain

${ }^{d}$ Centro Nacional de Aceleradores, E-41092 Sevilla, Spain

${ }^{e}$ Centre de Recherches du Cyclotron, Université Catholique de Louvain, B-1348 Louvain-la-Neuve, Belgium

${ }^{f}$ TRIUMF, V6T2A3 Vancouver B.C., Canada

${ }^{g}$ INFN Laboratori Nazionali del Sud and Sezione di Catania, I-95123 Catania, Italy

${ }^{h}$ Department of Physics, University of York, YO10 5DD Heslington, York, U.K.

${ }^{i}$ Dpto. de Física Atómica, Molecular y Nuclear, Universidad Complutense, E-28040, Madrid, Spain

${ }^{j}$ School of Physics and Astronomy, University of Birmingham, B15 2TT Birmingham, United Kingdom

${ }^{k}$ Institut for Fysik og Astronomi, Aarhus Universitet, Dk-8000 Aarhus C, Denmark

${ }^{l}$ Centro de Fisica Nuclear da Universidade de Lisboa, 1649-003 Lisbon, Portugal

${ }^{m}$ Van der Graaff Laboratory, Nuclear Research Centre, AEOI, PO Box 14155-1339, Tehran, Iran

${ }^{n}$ The Andrzej Soltan Institute for Nuclear Studies, 00-681 Warsaw, Poland

${ }^{o}$ Instituut voor Kern-en Stralingsfysica, University of Leuven, B-3001 Leuven, Belgium

\begin{abstract}
During the past ten years the present collaboration has carried out several experiments related with the study of radioactive nuclei. One of the topics in which we have centered our research, is the scattering of halo nuclei at energies around the Coulomb barrier. As part of this study, we present in this work a review of the results obtained from the scattering of ${ }^{6} \mathrm{He},{ }^{11} \mathrm{Be}$ and ${ }^{11} \mathrm{Li}$. The presence of a "halo" in these exotic nuclei is found to have a striking effect on the dynamics of these reactions, making their study an interesting experimental problem and a challenge for existing reaction theories.
\end{abstract}

Keywords: Halo nuclei, elastic scattering, breakup products

PACS: 24.10.Eq, 24.50.+g, 25.45.De, 25.60.Gc.

\section{INTRODUCTION}

The increasing number of experiments performed at many facilities around the world has contributed to unveil the properties of exotic nuclei. Among these remarkable nuclei we find the so-called "halo nuclei" that are characterized by a very unusual structure, consisting of two distinct parts: a central core, usually

Application of Accelerators in Research and Industry

AIP Conf. Proc. 1336, 570-572 (2011); doi: 10.1063/1.3586166

(C) 2011 American Institute of Physics 978-0-7354-0891-3/\$30.00 
composed by a stable nuclei, and a halo of neutrons or protons orbiting at a large distance of the central core [1]. Therefore, a common property of the halo nuclei is their large nuclear radius. Moreover, the halo nucleons are weakly bound to the core, giving rise to a large dissociation probability in reactions with other nuclei, even at energies below the Coulomb barrier. In this work we will show part of our results related with the study of the dynamics of the halo nuclei ${ }^{6} \mathrm{He},{ }^{11} \mathrm{Be}$ and ${ }^{11} \mathrm{Li}$ by heavy targets at Coulomb barrier energies. All of them are neutron halo nuclei and, in the case of ${ }^{11} \mathrm{Li}$ and ${ }^{6} \mathrm{He}$, they are examples of Borromean nuclei [2] (i.e. three-body systems where the two-body subsystems are unbound). Regarding ${ }^{11} \mathrm{Be}$, it is a very weakly-bound one-neutron halo nucleus with a deformed ${ }^{10}$ Be core.

\section{EXPERIMENTAL SETUPS}

The setups for the referred experiments, present some similarities and differences. A common feature is the kind of detectors used. In general we used strip detectors with different shapes, according with the requirements of each measurement. To obtain mass and charge separation, we normally built telescopes in order to employ the energy loss technique. Using small telescopes we can ensemble complex geometries to cover specific angular ranges, to finally obtain differential cross sections (i.e. elastic, breakup, etc) for each beam and energy [3].

\section{SPECTROSCOPIC ANALYSIS}

Once the data related with each experiment are stored, we start a spectroscopic analysis, normally generating spectra of one and two dimensions, where the important variable is the energy signal registered in the components of each telescope. If we plot the energy registered in the $\Delta E$-detector as a function of the total energy (i.e. $\mathrm{E}+\Delta \mathrm{E}$ ) we can obtain spectra which allow the separation of the different isotopes produced from the projectile-target interaction. Once the reaction channels of interest are identified, we proceed to isolate and integrate them, in order to obtain the experimental associated cross sections.

\section{RELEVANT RESULTS}

We have performed two experiments at the CRCLouvain-la-Neuve facility in which the elastic scattering of ${ }^{6} \mathrm{He}$ by ${ }^{208} \mathrm{~Pb}$ was measured, for different energies around the barrier [4,5], as well as the ${ }^{4} \mathrm{He}$ products coming from this reaction [6]. In Fig. 1a, we show the measured elastic cross section $\left(E_{l a b}=22\right.$ $\mathrm{MeV}$ ) along with theoretical calculations, namely, an optical model fit of the data, and three-body and fourbody CDCC calculations [7,8].

In the case of the ${ }^{11} \mathrm{Be}$, we carried out one experiment using a beam at $32 \mathrm{MeV}$ at the REXISOLDE-CERN facility, Geneva [9], obtaining experimental results about the quasi-elastic scattering on a ${ }^{120} \mathrm{Sn}$ target. At the same time, we registered ${ }^{10} \mathrm{Be}$ as reaction fragment produced from the interaction of the ${ }^{11} \mathrm{Be}$ projectiles with the target. The quasi-elastic measurements are compared in Fig. 1b, with coupledchannel and CDCC calculations. More results from this campaign of experiments can be consulted in Ref. [10].

In a more recent experiment performed at TRIUMF Vancouver facility, we measured the scattering of ${ }^{9,11} \mathrm{Li}$ on ${ }^{208} \mathrm{~Pb}$ at two different energies, again at Coulomb barrier. The analysis of the obtained data is in progress. Some preliminary results can be found in Fig. 1c, where also are included theoretical interpretations based in a semi-classical model and CDCC calculations [5,7].

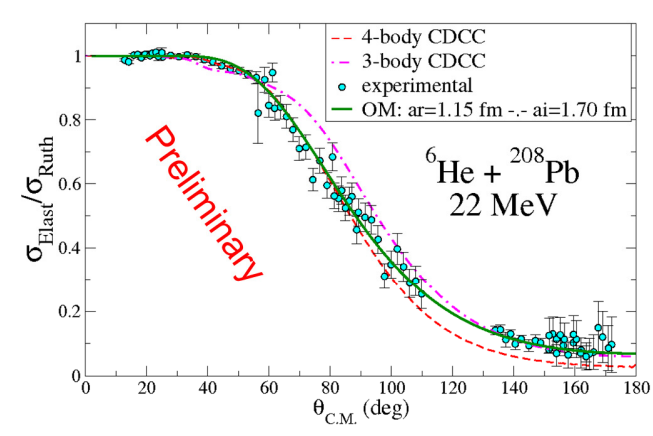

(a)

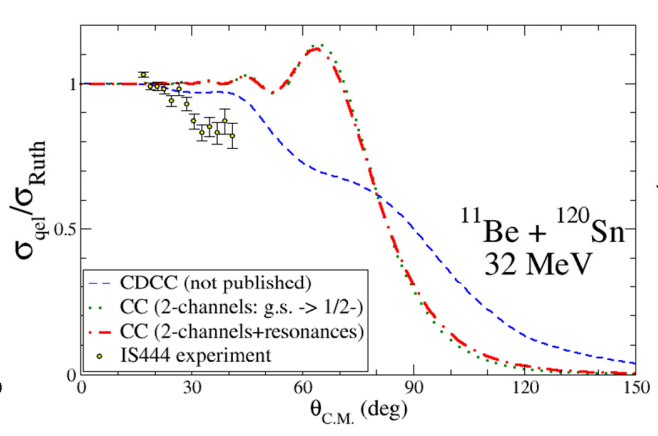

(b)

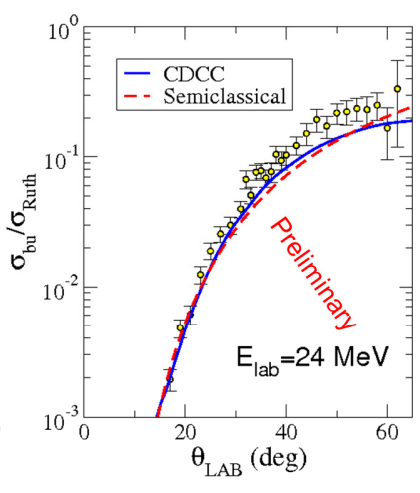

(c)

FIGURE 1. Some results from the reaction channels studied and theoretical calculations to describe them. (a) Elastic scattering of ${ }^{6} \mathrm{He}$ on ${ }^{208} \mathrm{~Pb}$ at $22 \mathrm{MeV}$, results from [5] forward and backward angles and "preliminary" results from PH215 Experiment at angles around $90^{\circ}$ [3]. (b) Quasi-elastic scattering from ${ }^{11} \mathrm{Be}$ on ${ }^{120} \mathrm{Sn}$ at $32 \mathrm{MeV}$ taken from [9]. (c) Preliminary results from the breakup probability of ${ }^{11} \mathrm{Li}+{ }^{208} \mathrm{~Pb}$ reaction at $24 \mathrm{MeV}$. 


\section{CONCLUSIONS}

From this extended study about the scattering of halo nuclei around the Coulomb barrier it is relevant to mention that, the behavior of the cross section is remarkably different from that of stable nuclei. The elastic angular distribution departs significantly from the Fresnel-like behavior which characterizes the scattering of heavy ions at Coulomb-barrier energies.

Furthermore, comparing our results with the typical Rutherford pattern, we could find a lot of differences starting in the region of the nuclear rainbow [11]. In the case of ${ }^{6} \mathrm{He}$ at $22 \mathrm{MeV}$ where we have a wide angular distribution, it is easy to observe the discrepancies between halo and stable nuclei.

For the ${ }^{11} \mathrm{Be}$ data, the absorption process [12] appears even at very forward angles, and presently the collaboration is investigating its origin.

Finally, the first results obtained from the analysis of ${ }^{11} \mathrm{Li}$ show a strong probability of breakup reaction, which appears even at very forward angles.

\section{ACKNOWLEDGMENTS}

This work has been supported by the Spanish MCyT projects FPA2005-04460, FPA2005-02379, FPA-2000-1592-C03-02, FPA2003-05958, FPA200204181-C04-02/03, FPA2006-13807-c02-0, FPA200907653, by the Spanish Consolider-Ingenio 2010 Programme CPAN (CSD2007-00042), by the contract number HPRI-CT-1999-00110 and the Belgian program P5/07 of the Belgian-state Federal Services for Scientific, Technical and Cultural Affairs. L. Acosta wants to thank to the CPAN-postdoctoral program for the support granted to realize this work.

\section{REFERENCES}

1. P. Hansen, B. Jonson, Europhys. Lett 4, 409 (1987).

2. K. Katō, S. Aoyama, S. Mukai, K. Ikeda, Nucl. Phys. A 558, 29c-4c (1995).

3. L. Acosta, "Estudio de las reacciones inducidas por núcleos halo a energías cercanas a la barrera de Coulomb", Ph.D. Thesis, University of Huelva, 2009. http://hdl.handle.net/10272/2794 ISBN: 978-84-9294462-0 (2009).

4. O. Kakuee, et. al., Nucl. Phys. A 339728 (2003).

5. A. Sánchez-Benítez, et. al., Nucl. Phys. A 803 30-45 (2008).

6. D. Escrig, et. al., Nucl. Phys. A 792 02-017, (2007).

7. K. Rusek, I. Martel, J. Gómez-Camacho, A. Moro, R. Raabe, Phys. Rev. C 72. 037603 1-4 (2005).

8. M. Rodríguez-Gallardo, et. al., Phys. Rev. C 77 (064609) 1-9 (2008).

9. L. Acosta, et. al., Eur Phys. J. A 42 461-464 (2009).

10. A. Di Pietro, et al., Phys. Rev. Lett. (Accepted)

11. N. Keeley, et. al., Nucl. Phys. A 571 326, (1994).

12. E.F. Aguilera, et. al., Phys. Rev. C 63 (061603(R)) 1-4 (2001). 